Literature Review

\title{
Social determinants of non-communicable disease
}

\begin{abstract}
Background: Non-communicable diseases (NCDs) are noninfectious diseases that are not transmitted through personal contact, biological vectors, vehicles, and are not airborne. The major NCDs include diabetes, cardiovascular diseases (CVDs), cancer, chronic respiratory disease, injuries (accidents) and mental illness. These are the world's growing causes of preventable illness, disability, morbidity and mortality.
\end{abstract}

Objectives: This literature review study conducted for exploring the social determinants of Non-Communicable Disease.

Methods: A literature review was done by using a variety of search engines; a Boolean search strategy to retrieve research publications, "grey literature", and expert working group reports.

Key findings: This review paper tried to explain globalization and its impacts on NCDs, focusing on global and regional structural and intermediary determinants of health inequalities and disparities, human right, gender and relevance of human right based approach (HRBA) on NCDs, Sustainable Development Goals (2015-2030) which are post 2015 (MDGs) and others.

Conclusion: The paper focused on human development; by discussing the components of SDGs, and the social, economic, and environmental domains that affect its achievement. NCDs are embedded in the post-2015 development agenda, since they are leading causes of death and disability, through their effects on the societal, economic, and environmental domains that affects negatively to the sustainability of human development. Some drives of unsustainable development, such as the transport, food and agriculture, and energy sectors, also increase the risk of NCDs.

Keywords: non-communicable disease, cardiovascular, globalization, urbanization, demographic
Volume 8 Issue 4 - 2019

\author{
Elias Teages Adgoy \\ Department of Community Medicine and Primary Health Care, \\ Orotta College of Medicine and Health Sciences, Eritrea
}

Correspondence: Elias Teages Adgoy, Department of Community Medicine and Primary Health Care, Lecturer II

Orotta College of Medicine and Health Sciences, Eritrea,Tel +29|-7285785, 29|-7|49024, Email eliasteaes@gmail.com

Received: May 14, 2019 | Published: August 23, 2019

\section{Introduction}

Non-communicable diseases (NCDs) are non infectious diseases that are not transmitted through personal contact, biological vectors, vehicles, and are not airborne. The major NCDs include diabetes, cardiovascular diseases (CVDs), cancer, chronic respiratory disease, injuries (accidents) and mental illness. These are the world's growing causes of preventable illness, disability, morbidity and mortality. The Millennium Development Goals (MDG) from 2000 to 2015 gave little focus for non-communicable diseases and their main social determinants tackling for the eight MDGs targets. Despite the evidences for the increased burden, non communicable diseases have ignored by health policy makers, multilateral and bilateral governmental and non-governmental organizations. Even though the causes and the threat that are posed by non-communicable diseases to health care systems are preventable, there have been inadequate national and global actions. The new Sustainable Development Goals agenda for the coming fifteen years from 2015 to 2030 identified different goals and targets for addressing the main non-communicable diseases and their determinants. This is one of the successful initials toward equity in health and well-being, through tackling the health inequities resulted by these determinants and setting the necessary preventative, curative and rehabilitative policies. Most targets are constructed based on human right and programs to address health problems associated with NCDs.

\section{Globalization and the impacts on NCDs}

One of the main determinants of NCDs is globalization. Globalization has direct and indirect effects to the individual, family, population, health care systems and national development. In many countries population increases and socio-economic transition have led to fast urbanization and expansion of cities in search of better livelihood opportunities. People with non-communicable diseases are increasing mainly due to population growth, demographic and nutritional transition, urbanization, and increasing obesity and decreased physical activity. By 2030, most of the world's population is expected to be concentrated in the urban areas. Major demographic changes and increased proportion of the age group above 65years will be seen globally ${ }^{1}$ such a change will increase the demand of infrastructure, services and the need for public places. Following such changes risk factors for NCDs in the socio-economic environment will become widespread. Subsides for agricultural products and fertilizers will help for the production of unhealthy agricultural products that will be easily available at low prices. Agricultural policies that were introduced for market liberalization gave a way for greater specialization in certain and specific crops in some countries to be readily available than other or variety of foods for consumers. ${ }^{2}$ Reducing import taxes such as import quotas usually lowers the cost of unhealthy food and food products such as fatty meats, soft and alcoholic drinks, and tobacco. Usually it is more difficult to reach to an agreement for governments to introduce policies and laws to control the supply and price of unhealthy food products. ${ }^{3}$ Majority of the cost and expenses of the social determinants of NCDs that include; education, health services, employment, social services and transport are covered by the revenue earned from different types of taxations.

One of the challenges for policy makers is banning of the production and marketing campaigns of the tobacco and alcohol 
industries Transnational tobacco companies deny the adverse effects of passive tobacco smoking, and aggressively exploiting the growth in tobacco sales mainly in developing countries. The alcohol industries are also globalized similarly to the tobacco industries and the role in causing non-communicable disease (injuries and violence) epidemics is complex. The main targets of these companies are women and young people. During the past years a remarkable change has been seen. Agricultural transition and transformation, and industrialization of farming, food processing and packaging, transportation and distribution, shopping, and the consumption of food outside of home were practiced. Change in the patterns of food production, processing and consumption can trigger the high incidence of non-communicable disease that threaten achievement of sustainable development goals. ${ }^{4}$ In most developed countries, about $75 \%$ of deaths are due to noncommunicable diseases that include; cardiovascular disease (CVD), malignancy, accidents or violence, whereas in developing countries NCDs are the most prevalent and common cause of death. Thus; it is believed that addressing the problems related with non-communicable diseases to reduce disability and death while and to increase health gains globally. ${ }^{5}$ Governments in many low and middle-income countries are now facing an increasing burden of deaths from injuries due to accidents, associated with increasing rates of urbanization and traffic injuries (Road Safety Training Manual, 2019). Nowadays injuries are the leading cause of death among young age group, people aged 15-29years. ${ }^{6}$

With an increase in economic development crude death rates from NCDs rises mainly in aged population. The age-standardized death rate is lower in high-income countries than in low and middle-income countries. Age-standardized NCD death rate is higher in men and in the areas of eastern and central Europe and central Asia, this death rates are due to high cardiovascular disease, whereas, in women death rates are found to be higher in South Asia which is mainly because of high cardiovascular and chronic respiratory disease . NCDs are as prevalent as $8 \%$ in sub-Saharan Africa and $85 \%$ in central and Eastern Europe and central Asia. The contribution of NCDs to the all-cause mortality in these countries is more than $50 \%$ in all regions. ${ }^{7}$

The best areas for health promotion activities for the prevention of NCDs are areas where people gather and these places include such as workplaces and schools. Policies should have to be set that inhibit direct use of tobacco, alcohol, unhealthy foods and drinks by imposing heavy taxation, reduction of production and restriction of advertisement and developing regulation regarding consumption which may have a positive effect on the prevention and control of NCD. Policies and regulation related to housing and shelter, employment, access to health and education, urban development policy, and social protection also contribute to the prevention and control of NCD. ${ }^{8}$ In Most countries people with low socioeconomic status have a higher risk of dying from non-communicable diseases, because of high smoking rates, blood pressure, and several other NCD risk factors. ${ }^{7}$ even though the use of tobacco has fallen in many high-income countries, but is rising rapidly in many low-income and middle-income countries with a prevalence of more than $25 \%$ in adolescents and still it accounts one in six of all deaths. This rise is due to the increases tobacco industries, uncontrolled activities and persistent efforts to influence and weaken tobacco control policies in the low and middle income countries. ${ }^{9}$

\section{Social determinants of health inequities associated with NCDs}

Social Determinants of Health inequities include; non-modifiable (age, genes, fetal origins) and modifiable determinants of health that include; behavioral risk factors (tobacco use, unhealthy food choices and obesity, physical inactivity, drug and alcohol use), effective health services (preventive, curative and rehabilitative health services, access and affordability of health services) and healthy environments (safe and supportive physical environment, good social-economic conditions, enough and quality food supply, healthy policy and governance, socio-cultural culture, societal value, limited access to tobacco, and alcohol). ${ }^{10}$

\section{Structural determinants NCDs}

Non communicable diseases are exaggerated by a large number of underlying causes that are embedded in the socio-economic, socio- cultural and political context of the country. Market forces, National government's policies and programs can limit NCDs by raising revenue, imposing heavily taxing on tobacco and other harmful substances producers and sellers. A successful action can be taken against tobacco use by an implementation of certain taxation and financial rules and regulations; legislations of certain policy interventions, establishment of indoor and outdoor public places, among businesses and employers, and use of media for promotion activities to change norms/behaviors regarding tobacco use, creation of smoke-free air laws that protect non-smokers from second-hand tobacco smoke. Policies and environmental approaches that built environment community, zoning standards for street connectivity, safe walking area, accessible road for disabled, people with impairment and children for cycling, and walking. ${ }^{11}$ This environment can be modified through dedicated recreation, exercise spaces, proximity to urban transport, good engineering of traffic, identification and improvement of dangerous sections of the road for the reduction of traffic-related injuries. The use of public information and different educational promotions are also the best way of the reduction for selfinjury. ${ }^{6}$ Aged people require long-term care needs than younger ones and these leads to an increase in the cost of expenditure by imposing an economic burden to their families and society. Now-a-days because of demographic transitions the burden of providing the needs of older people will fall on a smaller portion of the population. ${ }^{12}$

\section{Intermediary determinants of NCDs}

Health of an individual is determined by different social, economic and environmental circumstances of his surroundings, even though it influenced by the individual's characteristics and behavioral patterns (life style). The increase in the prevalence of NCDs is mainly associated with the modifiable behavioral risk factors that are compounded by often unorganized institutional arrangements to tackle these diseases and risks. ${ }^{6}$ Tobacco disproportionately affects the poor and the less educated, and it is considered as the single largest preventable risk factor. There are certain unconsidered causes of NCDs such as household air pollution from solid fuels lies between smoking and exposure to second-hand smoke in terms of its harm to health. ${ }^{6}$

Gender, age, ethnicity, religion and availability are also determinants of health inequalities. The study on the prevalence of non-communicable diseases in Eritrea, reported that tobacco use as the risk factor for most of the NCDs. Smoking in the general population in 2004, was reported as $8.1 \%$; with a prevalence of $15 \%$ among men, as compare $d$ to $0.6 \%$ among women. Prevalence rates were higher in those older than 45 years of age. The prevalence of tobacco smoking was higher among Muslims (11.4\%) than Orthodox Christians (5.8\%) and among alcohol drinkers $(10.2 \%)$ than nondrinkers $(6.6 \%)$. The majority of tobacco users $(89.3 \%)$ used commercially available cigarettes. $^{13}$ 


\section{Inequalities and disparities on NCDs}

Global inequalities in NCDs such as Benign Prostate Hypertrophy $(\mathrm{BPH})$ in males and breast cancers in females were recorded to be more prevalent in high-income than in low-income countries. Workers with NCDs have an increased risk likelihood of missing and underperforming at work, or becoming disabled. Most low and middle-income countries lack health insurance. Therefore, in such cases NCDs cause much greater economic losses. Women might go to work to become careers and children will be forced to leave school to supplement household incomes. There should be a law that protects children and young people through regulation, legislation, and market interventions. ${ }^{9}$

Cultural factors, social and health policies are the most important factors for the social gradient of a country at different stage of economic development. Social inequalities as a risk factor account for more than half of inequalities in NCDs. People in low-income and socioeconomic status have less access to quality health care services, for an urgent and timely diagnosis and treatment than in high-income countries or people with high socioeconomic status. Effective actions to reduce NCDs inequalities certain things should be included; such as early childhood development programs and education; securing an employment for disadvantaged group of people; comprehensive strategies for tobacco, alcohol and health food use and control. Universally acceptable, financially and physically accessible quality of primary care mainly on preventive and early detection and treatment of NCDs. ${ }^{7}$

\section{Human rights and NCDs \\ Relevance of HRBA NCDs}

Human right as a right is to live without discrimination of race, sex, political belief, religion, economic status and social condition. The right to enjoyment of a different facilities, goods, services and conditions that is necessary for attaining a standard health as the fundamental rights of every person. The UN High-Level Meeting on NCDs in September, 2011 provided an unrivalled opportunity to create a sustained rights-based global movement to tackle NCDs. ${ }^{14}$ Health is a fundamental human right which is integral to poverty reduction and development. The right to health is beyond the access to health care; the health policy of many countries addresses the needs of their population. But the human rights-based approach address the social determinants of NCDs in a practical way by including gender, equality, ethnicity, poverty, economic exclusion, non-discrimination and other socially determined barriers. The United Nations decision to tackle non-communicable diseases as a major part of its mandate is an international human dignity and human rights imperative. Equality and non-discrimination (women, children, indigenous and vulnerable populations), the right to information, education, participation, accountability, transparency, empowerment, sustainability and international cooperation; have particular application when examining a human rights-based approach in addressing NCDs. Throughout the world there are marked inequalities for indigenous people's because in majority the health status is worse than other population groups. For such people most of the human rights and fundamental freedoms of equal protection of law, physical, mental and moral integrity are absolutely crucial. A human rights-based approach to NCDs is concerned with the population groups most exposed to human rights violations that are mainly, women, children, aged, disabled and other vulnerable group of people that require a specific human right that guarantee special care, assistance and protection. ${ }^{14}$

\section{Gender and NCDs}

Majority of the NCDs and mainly diabetes and cancer weaken social interaction through stigma and discrimination. Women with diabetes usually face discrimination and stigma that leads to cultural pressure of non-exposing her disease that can create a barrier to diagnosis, treatment, employment, and marriage. NCDs prevent people from playing an active part in society. NCDs impact women in developing countries in their most productive years, affected by and contribute to gender inequality. The costs of NCDs are high and escalating, in terms of healthcare, lost productivity and social disruption. ${ }^{15}$ there is a growing concern about diseases that affect productivity and development of women and their families. The right to health, includes sexual and reproductive health, encompasses the freedom to control one's health and body as well. Breast cancer is treatable if it is detected early. Despite being largely treatable, it is the leading cause of cancer death in women, whereas, Lung cancer is the second leading cause of death for women. Cigarette smoking accounts for $50 \%$ of lung cancer cases in women. CVD is also the largest killer of women worldwide and it is increasingly impacting women in developing countries. Global alcohol consumption has increased in developing countries. As the treatment programs tend to be based on the needs of men, early detection and treatment of alcohol-related complications in women is limited and leaving them at risk of developing NCDs. Women are with limits on their ability to pay for healthcare for NCDs because they lack an access and control over resources. ${ }^{16}$ Women have a limited access to written information about NCDs and their risk factors, prevention and treatment because of a higher rate of illiteracy. In many places cultural taboos affect women's health seeking behaviors or need to medical care services from male health providers (Muslim fundamentalist countries). At the same time there is a shortage of female health professionals. Innovative partnerships are required to improve access to affordable, quality-assured, gender-sensitive essential medicines to treat NCDs in developing countries. Women's rights are still in need of being realized, and this lack impacts on the control and treatment of NCDs. ${ }^{14}$

\section{Analysis and discussion}

This review paper tried to explain globalization and its impacts on NCDs, focusing on global and regional structural and intermediary determinants of health inequalities and disparities, human right, gender and relevance of human right based approach (HRBA) on NCDs, Sustainable Development Goals (2015-2030) which are post 2015 (MDGs) and others.

\section{Conclusion}

The consequences of unplanned urbanization are the expansion of slums, poor housing and transportation systems; and inadequate energy services, absence of safe and adequate drinking water, improper sewage, and solid-waste management. The presence of NCDs can be also exacerbated by outdoor risks factors such as air pollution, poor physical activity, and injuries; from extremes of weather. The expansion of cities is usually associated with extensive and more service requirement due to extreme urbanization. Such problems were assessed in the MDGs and will be addressed by Goal 11 of SDGs" Make cities and human settlements inclusive, safe, resilient and sustainable". Because the SDGs agenda are built and prepared based on the gaps of MDGs, looking health as a core component for human development; in the post-2015 development agenda NCDs are considered as the leading causes of morbidity, disability and death. Some drives of unsustainable development, problem in transport, 
food production and agricultural activities and energy services, also increase the risk of NCDs. by 2030, NCDs total share of deaths is projected to increase by more than $50 \%$ mainly in low and middleincome countries. The magnitude of inequity that result health problems are greatest among poor people. ${ }^{15}$

Goal 3, of the sustainable Development Goals says, "Ensure healthy lives and promote well-being for all at all ages". So in this goal we have two key words "for all" that implies without discrimination of gender, ethnicity, indigenous peoples, marginalized people, religion, race, class, caste, political opinion, sexual orientation, vulnerable, and disability; and "at all ages " this goal also includes all age group ( from neonate to old age); here the specific target 3.4 and 3.6 of the Sustainable Development Goals, "By 2030, is proposed to reduce premature mortality from non-communicable diseases by one third, through prevention, treatment and promotion of mental health and well-being" and target 3.6 informed that "halve of the global deaths and injuries will be due to road traffic accidents by the year 2020;". SDGs consider the centrality of health, by improving food security and good nutrition because these are the basic foundations for sustainable development. The inclusions of NCDs are welcomed in the SDGs, because the burdens of NCDs in population demonstrate the interrelationship of different health challenges and development. ${ }^{17-21}$

\section{Acknowledgments}

None.

\section{Funding}

None

\section{Conflict of interest}

The author declares there is no conflict of interest.

\section{References}

1. Wild S, Roglic G, Green A, et al. Global prevalence of diabetes estimates for the year 2000 and projections for 2030. Diabetes Care. 2004;27(5):1047-1053

2. Hawkes C, Friel S, Lobstein T, et al. Linking agricultural policies with obesity and noncommunicable diseases: A new perspective for a globalizing world. Food Policy. 2012;37:343-353.

3. Legge D, Gleeson D, Snowdon W, et al. Trade Agreements and Noncommunicable Diseases in the Pacific Islands: School of Public Health and Human Biosciences. La Trobe University. 2013;1-16.

4. Beaglehole R, Yach D. Globalisation and the prevention and control of non-communicable disease: the neglected chronic diseases of adults. Lancet. 2003;362(9387):903-908
5. Nissinen A, Berrios X, Puska P. Community-based noncommunicable disease interventions: lessons from developed countries for developing ones. Bull World Health Organ. 2001;79:963-970.

6. Jamison DT, Summers LH, Alleyne G, et al. Global health 2035: a world converging within a generation. Lancet. 2013;382(9908):1898-1955.

7. DiCesare M, Khang YH,Asaria P, etal. Inequalities in non-communicable diseases and effective responses. Lancet. 2013;381(9866):585-597.

8. Clark H. NCDs: a challenge to sustainable human development. Lancet. 2013;381(9866):510-511.

9. Beaglehole R, Bonita R, Horton R, et al. Priority actions for the noncommunicable disease crisis. Lancet. 2011;377(9775):1438-1447.

10. Nutbeam D. Health literacy as a public health goal: a challenge for contemporary health education and communication strategy into the $21^{\mathrm{s}}$ century. Health Promotion International. 2006;15(3):259-267.

11. Bauer UE, Briss PA, Goodman RA, et al. Prevention of chronic disease in the 21st century: elimination of the leading preventable causes of premature death and disability in the USA. Lancet. 2014;384(9937):4552 .

12. Bloom DE, Chatterji S, Kowal P, et al. Macroeconomic implications of population ageing and selected policy responses. Lancet. 2015;385(9968):649-657.

13. Mufunda J, Debesay A, Mosazghi A, et al. Prevalence of tobacco use in Eritrea: Results from a non communicable disease risk factor survey. Nicotine Tob Res. 2007;9(7):777-779.

14. Human Right and the Social Determinants of Health NCDs and Human Rights. 2011.

15. Alleyne G, Binagwaho A, Haines A, et al. Embedding noncommunicable diseases in the post-2015 development agenda. Lancet. 2013;381(9866):566-574.

16. Bener A, Salameh KM, Yousafzai MT, et al. Pattern of Maternal Complications and Low Birth Weight: Associated Risk Factors among Highly Endogamous Women. ISRN Obstet Gynecol. 2012;540495.

17. UN-NGLS. WCRF International response to United Nations Non- Governmental Liaison Service consultation on the post-2015 development agenda planning process. UN-NGLS-ConsultationResponse; 2013.

18. https://www.who.int/mental_health/mhgap/mental_health_action_ plan_EN_27_08_12.pdf

19. Niaz A, Ali Z, Nayyar S, et al. Prevalence of NAFLD in Healthy and Young Male Individuals. ISRN Gastroenterol. 2011;363546.

20. Non Communicable Disease (NCDs). The Human Right Factor. The Lancet. 2011;377(9775):1438 -1447,

21. http://www.who.int/violence injury prevention/road traffic/activities/ roadsafety_training_manual_unit_2.pdf 